\title{
SOBRE EL «MANIFIESTO POR UNA PEDAGOGÍA POST-CRÍTICA»: PROBLEMAS DE TRADUCCIÓN A OTRAS TRADICIONES
}

\author{
On the "Manifesto for a post-critical pedagogy»: \\ translation problems into other traditions
}

\author{
Renato HUARTE CUÉLLAR \\ Universidad Nacional Autónoma de México. México. \\ renato@unam.mx \\ bttps://orcid.org/0000-0002-2419-4271
}

Fecha de recepción: 21/02/2020

Fecha de aceptación: 24/03/2020

Fecha de publicación en línea: 01/07/2020

RESUMEN

Hay diversas maneras de entender la traducción. Una de ellas tiene que ver con el aspecto de lo que se dice y cómo se dice en lenguas distintas. No obstante, como un problema filosófico, la traducción puede percibirse de diversas maneras y con implicaciones de diversa índole, vinculando el lenguaje con las formas de pensar, con las formas de actuar, entre otras. Desde esta perspectiva, se procurará ampliar este problema que ya identifican los autores en el propio Manifiesto en el sentido de dicho documento puede universalizarse. Si bien el Manifiesto busca dar cuenta, a partir de cinco principios, de la forma en la que nos podemos aproximar al fenómeno educativo, lo que se busca problematizar en este texto es la posibilidad de generalización del Manifiesto desde un contexto específico en el que ha sido escrito y pensado, digamos una tradición específica, y su posibilidad o imposibilidad de ser llevado hacia otras tradiciones o inclusive otros ámbitos de educación.

Si bien el Manifiesto es un documento importante ya que pone en tensión diferentes problemáticas que han sido abordadas desde lo que los autores denominan la tradición anglófona/analítica de filosofía de la educación, se pondrá en evidencia, usando como ejemplo, el hecho de analizarlo desde la filosofía de la educación en 
lengua española, lo cual es un problema de suyo, pero que vincula la forma en que esta pretensión de universalización puede ser un límite a la propia propuesta. Si bien lo que pretenden los autores del Manifiesto busca expresamente el diálogo, tal vez éste pueda ser repensado en otras lógicas o desde otras perspectivas que no habían sido pensadas, como es el contexto de la filosofía o el pensamiento en castellano y las implicaciones que tiene para el estudio de los fenómenos educativos.

Palabras clave: pensamiento crítico; pedagogía crítica; traducción; filosofía de la educación; pensamiento en castellano; tradiciones.

\section{ABSTRACT}

There are multiple ways in which translation can be understood. One of them has to do with what is said and how it is said in different languages. Nonetheless, as a philosophical problem translation can be perceived in different ways with numerous implications, binding language with the ways of thinking, ways of acting, among others. From this perspective, we would try to expand the problem already identified by the authors of the Manifesto: Can it be universalized? The Manifesto seeks to expose through five principles a way in which we can approach to education. In that sense, this article tries to question a possible universal aim from a specific context in which has been thought and written, a specific tradition, and the possibility to extend it to other traditions or other realms of education.

The Manifesto is an important document since it shows the tensions around different problems stated by the authors as being taken into account from the Anglophone/Analytical Philosophy of Education. Nonetheless, it will be shown, using the tradition of philosophy in Spanish speaking communities as an example, a problem within itself, if there could be a limit to the Manifesto proposal. The authors of the Manifesto state clearly a much broader dialogue. This might be rethought from other logics and perspectives not taken into account, just as philosophy in Spanish speaking contexts and its implications for understanding educational phenomena.

Key words: critical thinking; critical pedagogy; translation; philosophy of education; philosophy in Spanish; traditions.

\section{INTRODUCCIÓN}

Me gustaría comenzar por agradecer doblemente esta iniciativa. En primer lugar, realizar en lengua castellana un número monográfico sobre un fenómeno de relevancia en otra tradición ya implica abrir posibilidades de diálogo. En ese mismo sentido y, en segundo lugar, quiero agradecer el tono de diálogo del Manifiesto por una Pedagogía Post-Crítica, escrito y presentado en la Universidad de Liverpool 
Hope en Inglaterra en 2016 por Naomi Hodgson, Joris Vlieghe y Piotr Zamojski ${ }^{1}$. Si bien toda propuesta es y debe ser problematizada, más aún las filosóficas, este tipo de iniciativas deben abrir la posibilidad a ser miradas desde otras perspectivas y probadas de múltiples maneras.

¿Por qué un manifiesto? ¿Qué entienden los autores por post-crítico? ¿Qué sería, entonces, una perspectiva crítica, sobre todo en el ámbito de la filosofía de la educación? Como los autores declaran un manifiesto tiene que ser breve y tener declaraciones claras y concisas sobre algún asunto o programa específico. No se pretende en el presente trabajo dar cuenta de discusiones en torno a estas preguntas desde posturas en torno a la definición de conceptos específicos. Más bien se procurará dar cuenta de lo que implican estas preguntas para el mundo de la filosofía de la educación fuera del ámbito en donde estas preguntas fueron establecidas y respondidas, según los propios autores, en el mundo de la filosofía de la educación en el estilo anglófono y analítico, según reconocen los autores del Manifiesto. Esto es un buen punto de partida. Los autores mismos sostienen: "Al enunciar principios en la forma de un manifiesto, nos arriesgamos a ser acusados de universalismo, de normatividad excluyente». Ellos están conscientes de lo frágil que puede ser la forma de establecer un programa de análisis hacia donde el estudio de la educación deba tender. Sin embargo, un punto importante es que "hay» que tener principios y no permitir un desdibujamiento de los mismos a partir de la posmodernidad y el posestructuralismo.

Junto con este texto y el de otros colegas de distintas latitudes del mundo de habla hispana preocupados por los fenómenos educativos, será publicada una traducción del mismo Manifiesto en este mismo número monográfico. Realizar esta traducción, sin menospreciar la labor, no es el sentido de traducción al que haremos referencia. Más bien, partimos de una idea de traducción que remite a tradiciones distintas de filosofía de la educación. En ese sentido, ¿cómo se pueden traducir los postulados generales del Manifiesto más allá del contexto anglófono y de la filosofía analítica de la educación? ¿Cuáles son esos límites y alcances? ¿Qué dificultades aparecen al realizar este ejercicio? Para tal fin, utilizaremos lo que ha sido denominado de diferentes maneras que habrán de ser precisadas: "filosofía en español", "filosofía iberoamericana", "filosofía en Hispanoamérica", entre otras denominaciones posibles.

1. La versión española que se cita en este artículo forma parte del monográfico «Una respuesta iberoamericana al 'Manifiesto por una pedagogía post-crítica'»: http://dx.doi.org/10.14201/teri.22862 


\section{LA FILOSOFía de LA EDUCACIÓN DESDE EL PENSAMIENTO EN HiSPANOAMÉRICA COMO CONTEXTO}

Por la propia historia de la filosofía más extendida, tendemos a asumir la filosofía como hecha por hombres europeos en instituciones europeas, cuya cuna es la Grecia clásica y que, a través de Roma, llegó a la Europa medieval, renacentista y de épocas posteriores. Las lenguas de la filosofía, después de que Cicerón hiciera un acto de traducción de términos griegos al latín, se desarrollaría en lenguas europeas, ya hacia los siglos XVIII y XIX en inglés, francés o alemán. Esto no es una cuestión menor, ya que, salvo honrosas excepciones, inclusive otras lenguas europeas como el italiano o el castellano no figuraron en la historia de la filosofía como lenguas de referencia mayoritaria para el resto de los círculos filosóficos europeos. La mayoría de las historias de la filosofía presentan más bien la historia del pensamiento europeo que había llegado a diferentes partes del mundo (Copleston, 2011)².

Según expone Ángel Alonso (Huarte Cuéllar, 2018, pp. 81-98), el problema de la filosofía hispana está vinculada a una búsqueda de identidad que tiene su clímax en el siglo XX, proceso que no puede ser concebido de manera aislada del pensamiento de las antiguas colonias españolas en América (y tal vez en otros lados). Solo a manera de ejemplo, considero importante retomar el papel que tuvo José Gaos en este proceso. Este personaje realizó una crítica a esta forma de pensar la filosofía expuesta como lo hace Copleston. El autor, nacido en España y autodefinido "transterrado" (Zea, 2008) a México, fue el mayor impulsor de que en México y en América Latina en general, lo que él mismo denominaba Hispanoamérica, existe un pensamiento propio y no una mera apropiación de ideas de otras latitudes, retomando y criticando planteamientos de Unamuno y Ortega y Gasset, este último su profesor. Gaos trabajó distintos temas en el ámbito filosófico, además del problema de la filosofía en Hispanoamérica que claramente se derivaba de la reflexión en torno al contexto y origen social, cultural, político, lingüístico, etc. del pensamiento, muy probablemente dado este contexto que explica Leopoldo Zea sobre los españoles refugiados en otros países, incluido México, a los que el propio Gaos llamaba "transterrados». Se ve un continuum en donde el pensamiento español tendrá su correlato más con los países americanos, por su propia historia, que con otros. No obstante, la filosofía hispanoamericana para Gaos es mestiza, en el sentido de que es capaz de incorporar y realizar una lectura propia de otras tradiciones a la par que tiene una tradición propia (1943).

2. En su versión castellana es tal vez uno de los textos más consultados. Tiene 9 volúmenes. Resulta interesante que el título original es A History of Philosophy, título que podría haber sido traducido como Una historia de la filosofía y no como La historia de la filosofía. De cualquier forma, los títulos de cada volumen son representativos: t. 1 Grecia y Roma - t. 2. De San Agustín a Escoto - v. 2. t. 3. De Ockham a Suárez - t. 4. De Descartes a Leibniz - t. 5. De Hobbes a Hume - v. 3. t. 6. De Wolff a Kant - t. 7. De Fichte a Nietzsche - v. 4. t. 8. De Bentham a Russell - t. 9. De Maine de Birán a Sartre. 
Para Gaos, la filosofía no es mera teorización, sino también puesta en marcha, acción. Esto querría decir que el contexto que vivió Hispanoamérica sería distinto por las características históricas específicas de la región. En el ensayo El pensamiento hispanoamericano Gaos lo expone de la siguiente manera:

E1 "pensamiento» es aquel pensamiento que no tiene por fondo los objetos sistemáticos y transcendentes de la filosofía, sino objetos inmanentes, humanos, que por la propia naturaleza de las cosas, históricas, éstas, no se presentan como los eternos temas posibles de un sistema, sino como problemas de circunstancias, es decir, de las de lugar y tiempo más inmediatas, y, por lo mismo, como problemas de resolución urgente (...) (1943, p. 11).

De esta cita puede comentarse el hecho de que la filosofía no será igual en distintos espacios y contextos. Por ende, la filosofía hispanoamericana, puesto en términos del propio Gaos, tendrá otros referentes. En ese sentido, la filosofía da respuesta a su contexto. En él se encuentran las actividades más habituales y comunes y la reflexión y articulación de esa realidad tendrá una expresión filosófica distinta. Así pues, en Hispanoamérica no habrá grandes tratados o críticas (a la manera de, por ejemplo, Kant), sino más bien se privilegiará el ensayo, el artículo publicado en la prensa del momento, entre otras formas de escritura. Esto, en lo que refiere a las formas de escritura. Algo parecido sucederán con los temas filosóficos. Mientras que la filosofía «europea» trata sobre cuestiones metafísicas creyendo que éstas son la "filosofía primera", en el ámbito de la filosofía en Hispanoamérica se privilegiarán temas de filosofía política y filosofía de la educación, así como temas contingentes al espacio en el que habitamos los seres humanos de manera inmediata.

Para poder ejemplificar esto, me gustaría retomar las palabras de Ambrosio Velasco cuando refiere a la perspectiva que María del Carmen Rovira, una de las más dedicadas discípulas de José Gaos en lo que respecta a la filosofía mexicana, ha encontrado a lo largo de sus investigaciones en las últimas décadas:

Así pues, el enfoque metodológico de la obra [de Ma. del Carmen Rovira] está basado en dos premisas básicas: primero, que el pensamiento filosófico surge y se desarrolla en relación estrecha con la problemática social, política y cultural del momento y busca no solo comprender esa realidad, sino también intervenir en ella. Por ello con toda razón Carmen Rovira destaca la relación, política, ética y educativa del pensamiento político mexicano. En este sentido, coincide con José Gaos, quien considera que la filosofía hispanoamericana, lejos de reducirse a sistemas teóricos, como en el caso de la filosofía alemana, se caracteriza por ser ensayística, literaria, política y pedagógica. Pero Carmen Rovira y su grupo de investigadores, la mayoría de ellos jóvenes profesores que fueron sus alumnos, van más a fondo al ubicar como un segundo principio metodológico, el carácter polémico de la producción filosófica de cada autor en contextos intelectuales y políticos más complejo, esto es, en los «discursos». Se trata pues de analizar el pensamiento filosófico en toda su intensidad vital, atendiendo tanto a los argumentos racionales, como a los presupuestos y consecuencias ideológicas. (Rovira Gaspar, 2010, p. 11). 
Esto abre la posibilidad de repensar si entonces la filosofía realizada en contextos distintos a los hegemónicos debería ser abordada de otra manera, en donde la educación, la política, etc., sean los ejes de análisis. Además, como se ha indicado líneas arriba, esto permitiría dar cuenta de cómo realizar un acto de "traducción» de lo que se hace en otras tradiciones. Para tal fin, de manera muy sucinta, considero que entender las corrientes o tradiciones de filosofía de la educación que, desde Europa, han sido las más reconocidas daría el contexto final para poder hablar del Manifiesto.

De los estudios que se han hecho al respecto a las corrientes o tradiciones de filosofía de la educación vinculada a tradiciones de pedagogía y/o ciencias de la educación, el que he encontrado que brinda un panorama más amplio y detallado es La filosofía de la educación. Estado de la cuestión y líneas esenciales de Stella Maris Vázquez (2012, pp. 19-30) No me parece una coincidencia que esta reflexión venga de una autora argentina en el sentido que, desde Hispanoamérica (siguiendo lo establecido por Gaos) se haga una reflexión de las tradiciones (europeas) más comunes de filosofía de la educación.

La autora parte de la idea que debemos a la tradición anglosajona de filosofía el término "filosofía de la educación" per se. Sin embargo, para hacer un estudio de la filosofía de la educación en un sentido más amplio remite a tres tradiciones para articular no solo la discusión filosófica en torno a la educación, sino la propia concepción del campo disciplinario. Vázquez parte de un análisis epistemológico de las corrientes alemana, francesa y anglosajona. A continuación, daremos algunos visos de lo que considero lo más relevante del recorrido que hace Vázquez por las tres corrientes o tradiciones.

Lo que puede llamarse escuela alemana de filosofía de la educación, basados sobre todo en Dilthey y Spranger a partir de lo que se fue articulando en el siglo XIX sostendrán la pedagogía como ciencia cuyo objeto de estudio será el fenómeno educativo. (Vázquez, 2012, pp. 21-23). Dilthey ubicará a la pedagogía en las ciencias del espíritu como oposición a las ciencias de la naturaleza. De ahí, según Vázquez, se desprende una idea de la filosofía como fundamento de la explicación de los fenómenos a partir de la hermenéutica. Por otro lado, la escuela francesa retoma del positivismo del siglo XIX negando la posibilidad de una ciencia de la naturaleza que pretende la corriente alemana, sino que más bien existirán diversas ciencias que podrán abordar la educación como objeto de estudio. Vázquez ubica como personaje principal a Emile Durkheim. (Vázquez, 2012, pp. 21-23). En contraparte, es la escuela anglosajona tanto en su vertiente estadounidense con una predominancia pragmatista, así como en Gran Bretaña y Australia, con una predominancia de la filosofía analítica (Vázquez, 2012, pp. 19-20), que han tenido una evolución tanto a nivel disciplinario como gremial y explica la forma en que las asociaciones en los distintos países se conforman, de la John Dewey Society y su transición en 
a la Philosophy of Education Society (PES) en el Reino Unido, así como las otras asociaciones tanto en Estados Unidos como en Australia.

No es casualidad que, a partir del libro más famoso de John Dewey ${ }^{3}$, se hayan encauzado grandes esfuerzos académicos para desarrollar el área que, desde distintas corrientes de filosofía, daban sustento a un estudio contextualizado de la educación, la filosofía de la educación. Eso explicaría por qué un buen porcentaje de los libros sobre el tema provengan de esa raigambre. Muchas de las obras traducidas al castellano corresponden a autores de la tradición anglosajona, solo por el número de publicaciones en esa lengua.

Resulta sin duda interesante ver la interacción de estas tres corrientes entre ellas. Más aún sería relevante ver cómo otros espacios en donde se hace filosofía de la educación, teoría de la educación, pedagogía o como se quiera denominar este quehacer de reflexión y acción en torno al fenómeno educativo reaccionan a lo que se hace en estas tres corrientes mayoritarias. Sirva lo expuesto hasta este momento para dar contexto al objetivo del presente escrito: dar cuenta de algunos de los cuestionamientos posibles del Manifiesto en la tradición de lo que podemos llamar Hispanoamérica.

\section{3. «Traducir» el Manifiesto por una Pedagogía Post-Crítica al español: CUESTIONAMIENTOS Y REFLEXIONES}

Para poder articular estos cuestionamientos y reflexiones partiremos de tres puntos que considero son los más problemáticos o que, por lo menos, requerirían prestar atención de manera más cautelosa al emprender esta tarea de traducir lingüística y culturalmente el Manifiesto. Éstos son la discusión en torno a la forma de exponer las ideas como un manifiesto; la noción que se tiene de crítica y, en ese sentido, de post-crítica y, finalmente, la noción de los conceptos educación y pedagogía empleados en el texto; todo esto, en el sentido de las implicaciones que tendría este acto dialógico del estudio de los fenómenos educativos.

No creo que sea casual que estos tres elementos sean los que integran los principales conceptos o palabras del título. Sin embargo, tampoco considero que al abordar estos tres elementos se esté minando completamente las pretensiones del Manifiesto. Más bien, considero que son las discusiones nodales, en términos generales, así como las de mayor importancia en lo que respecta a su discusión en lengua española.

3. Recordemos que la "obra cumbre» de John Dewey se llama Democracia y educación, pero que lleva por subtítulo Una introducción a la filosofía de la educación. 


\subsection{Sobre escribir un Manifiesto}

Como se ha expresado al comienzo del presente texto, ya Naomi Hodgson, Joris Vlieghe y Piotr Zamojski reconocen que escribir a la manera de un manifiesto puede llegar a ser problemático por el hecho de que, sin llegar a ser una forma de exposición de ideas que busque argumentar académicamente sus principios, sí busca ser corto y exponer un mensaje contundente enarbolando un programa. Tal vez en nuestras latitudes, el manifiesto más famoso sea el escrito por Karl Marx y Friedrich Engels en donde se establecía el programa del partido comunista. Sin embargo, no viene a mi mente otro manifiesto así de famoso, por lo menos no he encontrado que en el mundo de la filosofía de la educación exista.

No obstante, una de las réplicas que en su versión inglesa acompaña al propio Manifiesto arroja luz para poder entender el origen de la asunción de este texto como un manifiesto. Se trata del caso del artículo de Tyson E. Lewis que lleva por título "A Response to the 'Manifesto for a Post-Critical Pedagogy'" (Hodgson, et al., 2017, pp. 23-34). En esta respuesta o réplica, Lewis pone en tensión la noción de manifiesto. Da cuenta de que los manifiestos tienden a tener una connotación colectiva, esbozar un programa claro con puntos precisos y concisos, así como estar vinculados a instituciones u organizaciones sociales o políticas. Esto es lo que el Manifiesto del Partido Comunista de Marx y Engels buscaba (p. 28). Claramente, dice Lewis, Hodgson, Vlieghe y Zamojski no buscan eso. Su propósito va más allá. Para efectos de lo que nos ocupa, Lewis va más allá y entonces da cuenta del contexto en el que un manifiesto en torno a la educación tiene sentido.

En esta búsqueda histórica, Lewis encuentra que el Manifiesto en cuestión entra en debate y confrontación con dos manifiestos anteriores que sí tienen su incumbencia en el mundo de la educación. Se trata de «When Schools Become Deadzones of the Imagination: A Critical Pedagogy Manifesto" de Henry Giroux, publicado en 2009, y "A Revolutionary Critical Pedagogy Manifesto for the Twenty First Century" de Peter McLaren, publicado en 2013. Sobre ellos, comenta Lewis que, en ambos casos, hay un tono profético que vaticina un futuro económico y social determinado sobre el cual es necesario actuar. Hodgson, Vlieghe y Zamojski se alejan de este tipo de postulado. Sin embargo, lo hace también como un manifiesto.

Teniendo este contexto, es interesante que ambos textos son publicados por un estadounidense y un canadiense. ¿Cómo se articularía esto en los países de habla hispana? Sin mucho temor a equivocarme, afirmaría que el papel de autores como Giroux y McLaren no sería tan central como en otras tradiciones. Tal vez inspirados en Marx, estos autores tratarán de articular esta postura emancipatoria con manifiestos, cuyos ecos resuenen a las tradiciones marxistas o neomarxistas, con todo un cometido. La conexión con el mundo de habla hispana no se da de manera directa, salvo por la relación directa con Paulo Freire. Este personaje latinoamericano, brasileño que vivió en Chile durante varios años y cuyo pensamiento influirá de manera muy particular la región latinoamericana (Kohan, 2019) tenderá 
lazos con McLaren y con Giroux. Baste como ejemplo la introducción al libro de Giroux Los profesores como intelectuales. Hacia una pedagogía crítica del aprendizaje elaborada por el propio Freire, que permite ver la relación que encuentra el educador brasileño con la postura del canadiense (1990). Esta relación ameritaría un acercamiento más cuidadoso. Sin embargo, aún en este caso, el Manifiesto sería una cuestión que, al menos en Hispanoamérica, no sería un género en el que los pensadores del fenómeno educativo utilizarían. La pretensión del texto de Hodgson, Vlieghe y Zamojski, al presentarse como un manifiesto, no solo se inscribe en una tradición con las implicaciones que estos tres autores y Lewis mismo en su réplica identifican como problemático, sino que además es un género que, para la tradición hispanoamericana, si es que la hubiera, resultaría extraño. Baste mencionar, solo a manera de ejemplo, tres autores de ambos lados del Atlántico. José Ortega y Gasset preferirá el ensayo o el artículo en el periódico (Bárcena, 1983), Domingo Faustino Sarmiento los discursos (Ibarra, 1985) y José Vasconcelos todas las formas de expresión anteriores (1998).

El reto para poder entablar una discusión con un manifiesto, cualquiera que fuera éste, sería establecer las pretensiones del hacerlo. Los tres autores del Manifiesto que nos convoca tienen una claridad que expresan mínima pero certeramente. El gran cuestionamiento es cómo entra en relación la lengua-cultura de recepción con un género de escritura con ciertas pretensiones en un contexto en donde esto no es tan natural. Tal vez esa pretensión que anuncian los tres autores de no querer universalizar sea traicionada no por los autores sino por nosotros los traductores.

\subsection{La crítica y la post-crítica}

Vinculado con lo expuesto en el apartado anterior sobre el Manifiesto, está la idea de lo que los autores del Manifiesto están entendiendo como "crítica" y "postcrítica». Si ya Lewis ha aclarado las pretensiones del manifiesto y su vínculo con la que se ha denominado "pedagogía crítica", entonces es menester ahora de entender el concepto de crítica y revisar la pretensión de anunciar una era "post».

Hablar de crítica en el mundo de habla hispana, sobre todo en los círculos académicos, tal vez deban remitirse a las interpretaciones que se hicieron de la obra de Karl Marx a lo largo del siglo XIX y, sobre todo, del siglo XX, siendo la obra de Max Horkheimer de 1937 Teoría tradicional y teoría crítica (2000) en el contexto programático de la Escuela de Frankfurt y la dispersión de sus miembros debido a las implicaciones de las leyes del nacional socialismo. Curiosamente la Escuela se instalará en Nueva York y tendrá una vida importante todavía hasta 1950 en esa ciudad norteamericana.

Del mundo de habla hispana, me gustaría traer a colación dos ejemplos para poder entender este valor de la crítica. El primero es el caso de Jesús Aguirre, quien sería el director literario de la editorial Taurus en Madrid, además de miembro de la Real Academia Española. Según ha mostrado Beatriz Caballero Rodríguez (2013, pp. 
338-345), el interés especial que guardaba Aguirre por la Escuela de Frankfurt, en especial por la obra de Walter Benjamin, llevaría a ser un estandarte por el estudio de esa corriente de interpretación marxista en lengua española. Revisar detalladamente el impacto de las obras literarias de la Escuela de Frankfurt en España ameritaría un trabajo mucho más específico, mismo que puede ser rastreado en la mencionada obra de Caballero Rodríguez. Ella muestra la forma en la que estas ideas fueron retomadas por intelectuales españoles, muchos de ellos, como Aguirre mismo, desde perspectivas católicas que pueden ser leídas como poco ortodoxas.

El otro ejemplo que me gustaría traer a colación es el de los estudiosos de la tradición crítica en América, por mor del argumento, en especial a Adolfo Sánchez Vázquez y Bolívar Echeverría, ambos refugiados de España y Ecuador respectivamente en México. Stephan Gandler logra mostrar puntos de tensión a la vez que identificar vetas innovadoras en el pensamiento de estos dos mexicanos por migración. No solo a partir de la Escuela de Frankfurt, pero también como una posibilidad crítica, ambos autores hacen un estudio cuidadoso de aspectos de la tradición marxista. De Bolívar Echeverría rescata la posibilidad y coherencia de la teoría marxista de no ser un pensamiento eurocéntrico, sino más bien que el marxismo tiene, a partir de los contextos particulares, una gran veta de exploración filosófica y política en regiones del mundo como América Latina. (Gandler, 2007). En lo que respecta a Sánchez Vázquez, Gandler rescatará la posibilidad de entender a un Marx, sobre todo en sus años de juventud, mucho más potente en lo que respecta a la idea de praxis en distintas áreas de la filosofía, en especial la estética. Esto devuelve una lectura muy poco dogmática de Marx y permite entenderla en una dimensión mucho más promisoria para el siglo XXI.

Ninguno de los autores en cuestión vio la posibilidad de una cuestión "post» crítica. Más aún, el propio Sánchez Vázquez (2018) permitirá entender que cualquier postura filosófica no caduca en tanto entra en diálogo con otras personas y contextos de manera puntual y específica, sea Horkheimer en 1937 o los pueblos indígenas en América en pleno siglo XX. Los tres autores del Manifiesto que nos ocupa parecen entender el "post» como una forma de respuesta a las consecuencias que la llamada "pedagogía crítica" han tenido en los espacios educativos y los ámbitos de estudio en torno a los agentes y espacios educativos. Considero también que tal vez la pretensión de dicho Manifiesto deba estar enfocado de manera puntual hacia autores como McLaren o Giroux y no pretender dejar atrás a la teoría crítica como un todo. Me parece que la "crítica» en ese sentido es mucho más amplia y la recepción y aportaciones en el mundo hispano pueden ser mucho más complejas e interesantes.

\subsection{La propuesta de educación y pedagogía}

Finalmente, queda abordar el problema de la traducción de la forma en la que los autores del Manifiesto han entendido educación y pedagogía. Dejemos de lado 
esta idea de "pedagogía crítica" en el sentido que McLaren, Giroux y Freire realizan. De mi lectura, no hay una especificidad de lo que entienden por la palabra "pedagogía» y cómo se vincula con lo «educativo». Tengo la impresión, a reserva de hacer un estudio cuidadoso, de que ambas palabras tienen una implicación de espacios, actores, instituciones, etc., educativas sobre las cuales buscan realizar una crítica. Así, a manera de ejemplo, en La pedagogía del oprimido (1979) encuentro que Freire utiliza "pedagogía» en el sentido de un sistema con personas e instituciones específicas que mantienen una condición social y económica a nivel mundial. Sin embargo, no es clara la relación entre esa pedagogía y procesos educativos (¿pseudoeducativos?) y su distinción en prácticas concretas. Tal vez ese no sea el objetivo de la obra freiriana.

En el propio Manifiesto de Hodgson, Vlieghe y Zamojski puedo identificar varias formas de entender lo "pedagógico» y lo «educativo». Para empezar, cuando los autores describen el cuarto principio dicen lo siguiente:

Por muy tradicional o conservador que todo esto pueda sonar, deseamos defender la educación por el bien de la educación misma: la educación como el estudio de y la iniciación en una materia por su valor intrínseco y educativo, más que instrumental, de manera que pueda ser retomada de nuevo por la nueva generación.

Agregado a lo anterior, los autores incorporan, como se ha mostrado líneas arriba, la idea de "pedagogía crítica" sin hacer ninguna distinción de la forma en la que entienden la pedagogía que no sea tal. Más adelante, en el quinto y último principio, los autores hablan del «pedagogo» y es utilizado, a mi entender, de manera equitativa con el educador y con el profesor de escuela. Considero que aquí hay un problema que tiene que ver, nuevamente, con las tradiciones de filosofía de la educación como hemos procurado mostrar en el contexto.

Para comenzar con la tradición hispana, traigo a colación a Lorenzo Luzuriaga, otro español que, tras la Guerra Civil, decide ir a radicar a Argentina. Ahí será de los mayores impulsores de la traducción de textos pedagógicos de autores como Kant, Dewey, Herbart, Pestalozzi y Dilthey de las lenguas originales al español en la editorial Losada, así como profesor universitario en la Universidad de Buenos Aires. En su libro Pedagogía (1963) dará un panorama amplio de la educación como las formas culturales que tienen y han tenido los seres humanos para transmitir y reconfigurar a los individuos y a las sociedades. El término educación, si bien es polisémico, justamente podrá significar una multiplicidad de fenómenos, actores, instituciones, procedimientos con esta finalidad. En ningún momento esto lo circunscribe al ámbito escolar, si bien, en el siglo XX la escuela es la institución que ha sido privilegiada como el espacio educativo por excelencia. No obstante, corresponde a la pedagogía, como ciencia de la educación (Luzuriaga, 1963, p. 18), estudiar todas las formas contemporáneas y pasadas de educación, así como intervenir en el desarrollo de las formas futuras. Es probable que el autor tenga una influencia marcada de los autores, sobre todo alemanes, que distinguían la ciencia, 
RENATO HUARTE CUÉLLAR

SOBRE EL “MANIFIESTO POR UNA PEDAGOGÍA POST-CRÍTICA»: PROBLEMAS DE TRADUCCIÓN A OTRAS TRADICIONES

la pedagogía, de su objeto de estudio, la educación, no sin identificar una serie de problemas. (Luzuriaga, 1963, p. 20) ¿Por qué, entonces, los autores del Manifiesto en cuestión parecen no distinguir? La respuesta puede estar en lo que sostiene Enrique Moreno y de los Arcos, un pedagogo mexicano y profesor de la Universidad Nacional Autónoma de México, quien sostenía lo siguiente:

Quienes han propuesto sustituir la palabra pedagogía por la noción de ciencias de la educación o — más bárbaramente- por educación solamente (a la usanza anglosajona) ignoran que nuestro término tiene una larga tradición no solo en castellano sino en griego, italiano, portugués, francés, alemán, y ruso, por citar unos cuantos ejemplos. En todos estos idiomas es perfectamente clara la distinción entre la disciplina, pedagogía, y el fenómeno que estudia, la educación.

Las dudas en torno a la corrección de nuestro vocablo suelen surgir cuando, directamente o a través de traducciones, se leen obras pedagógicas de lengua inglesa. Se produce entonces un fenómeno en el que encuentro mucho de ignorancia, negligencia y de sumisión servil: no hay coincidencia de términos, pero el inglés no puede equivocarse, tenemos que ser nosotros los errados. Si en inglés no es usual la distinción entre la ciencia y el fenómeno, sino que se designan ambos con el mismo término, education, debemos todas las demás lenguas cultas adoptar tal modalidad. (1999, p. 11).

Esta forma de aproximarse al fenómeno educativo pretende, al nombrar, distinguir y encontrar respuestas a las problemáticas del mundo de la educación en una intersección de historia, filosofía, teoría de un fenómeno con la ayuda de otras disciplinas como la psicología, la política, la sociología, entre otras. Ni el pedagogo es necesariamente el educador, ni tampoco toda discusión sobre problemas educativos deben estar ceñidos a la escuela, una institución educativa que, en el pasar de los años a nivel histórico, no son sino un muy breve instante de la historia de la humanidad.

Puede entenderse, entonces, que los ejemplos del Manifiesto en cuestión busquen dar respuesta a lo que parecen ser problemas actuales e inmediatos como el profesor en el aula y que no distingan las funciones, como tal vez sea herencia de la tradición alemana a la que refería Vázquez, del educador de otras como el que realiza investigación sobre el fenómeno educativo, quien elabora políticas para la educación, etc. El problema no es de la tradición anglosajona desde donde está pensada, sino de cómo se interactúa desde otras tradiciones que pueden llegar a distinguir, por ejemplo, entre una disciplina y un objeto de estudio que, además, no solo se ciñe a lo escolar o a la educación familiar.

\section{A MANERA de CONCLUSIÓN}

Hemos tratado de mostrar, a lo largo de tres aspectos puntuales, ciertas posibles tensiones de pensar el Manifiesto de Hodgson, Vlieghe y Zamojski desde el pensamiento en español, de una tradición hispana, asumiendo que Hispanoamérica tiene ciertos rasgos al pensar cualquier cosa, en este caso la educación, desde lo 
que el castellano le permite. Es probable que esta tradición no sea tal. Sin embargo, considero que estas tensiones o advertencias en tradiciones de pensamiento que no son homogéneas pueden permitir que el diálogo se dé de mejor manera, entendiendo los límites y alcances de una propuesta a partir de su propio contexto hacia el contexto de otras tradiciones.

Hay muchos aspectos del Manifiesto que podrían ser discutidas sin tener esta discusión. Los autores van citando a autores que se han estudiado en lengua española. Tal es el caso de Arendt, Rancière, Badiou, Platón, entre otros. Otros más, desde mi experiencia, han sido trabajado en mucho menor medida por autores en lengua española. Tal es el caso de Cavell, Berlant, Emerson, Latour o Haraway. Esto, más que un problema, es una gran oportunidad. Entender la propuesta del Manifiesto, suponiendo que la pretensión de universalización ha quedado zanjada, es también la posibilidad de entender a autores de otra tradición que no se conocen en la propia. Cabría entonces llevar a cabo una propuesta inversa y que la otra tradición conozca no solo a los que hemos considerado "nuestros» autores, sino también «nuestras» formas de escritura, condiciones de estudio del fenómeno educativo, espacios y actores educativos concretos que podrían ampliar dicho panorama, etc.

El problema tratado en el Manifiesto de la "pedagogía hermenéutica" no ha sido abordada, sobre todo, por una cuestión de extensión de los trabajos. Esto convendría también ser trabajado, sobre todo en el contexto de la pregunta si es que en todas las tradiciones de pensamiento la pos-modernidad es un asunto relevante y, de serlo, en qué medida. Este tipo de preguntas son las que podrían permitir un diálogo más fructífero, a mi parecer.

Tampoco puede entenderse la noción de tradición en la filosofía de la educación de manera hermética (Huarte Cuéllar, 2018, pp. 9-19). Esto dependerá de las condiciones que cada una de las que se consideren tradiciones establezcan de manera consciente o inconsciente. La labor, entonces, queda abierta a poder aproximarse a esta propuesta como una más que conviene ser conocida, estudiada, rebatida y analizada con cuidado.

\section{REFERENCIAS BIBLIOGRÁFICAS}

Bárcena, F. (1983). La dimensión educativa del problema de la verdad en el pensamiento de José Ortega y Gasset. Revista Española de Pedagogía, 41(160), 311-324.

Caballero Rodríguez, B. (2013). Against Instrumental Reason: NeoMarxism and Spirituality in the Thought of José Luis L. Aranguren and Jesús Aguirre. Madrid: Anagnórisis.

Copleston, F. (2011). Historia de la filosofía. Barcelona: Planeta/Ariel.

Freire, P. (1979). Pedagogía del oprimido. México: Siglo XXI. 23. ${ }^{a}$ ed.

Gaos, J. (1943). El pensamiento hispanoamericano. México: El Colegio de México.

Gandler S. (2007). Marxismo crítico en México: Adolfo Sánchez Vázquez y Bolívar Echeverría. México: Fondo de Cultura Económica. 
Giroux, H. (1990). Los profesores como intelectuales. Hacia una pedagogía crítica del aprendizaje. Barcelona: Paidós/Ministerio de Educación y Cultura. 7. a ed.

Hodgson, N., Vlieghe, J. y Zamojski, P. (2017). Manifesto for a Post-critical Pedagogy. London: Punctum Books. Recuperado de: https://punctumbooks.com/titles/manifesto-for-a-postcritical-pedagogy/ (Consultado el 01/03/2020).

Horkheimer, T. (2000). Teoría tradicional y teoría crítica. Barcelona: Paidós Ibérica.

Huarte Cuéllar, R. (2018). La filosofía de la educación y sus tradiciones. México: UNAM.

Ibarra, A. C. (1985). Doce textos argentinos sobre educación. México: SEP/ El Caballito.

Kohan, W. (2019). Paulo Freire Uma biografía filosófica. Mais do que nunca. Belo Horizonte: Vestígio.

Luzuriaga, L. (1963). Pedagogía. Buenos Aires: Losada. 7. ${ }^{\text {e ed. }}$

Moreno y de los Arcos, E. (1999). Pedagogía y ciencias de la educación. México: UNAM. 2. ${ }^{a}$ ed.

Sánchez Vázquez, A. (2018). El joven Marx. Los manuscritos de 1844. México: UNAM/Ítaca. $3 .^{\mathrm{a}}$ ed.

Rovira Gaspar, M. C. (Coord.). (2010). Una aproximación a la historia de las ideas filosóficas en México. Querétaro: Universidad Autónoma de Querétaro.

Vasconcelos, J. (1998). Hombre, educador y candidato. México: UNAM.

Vázquez, S. M. (2012). La filosofía de la educación: estado de la cuestión y líneas esenciales. Buenos Aires: CIAFIC Ediciones.

Zea, L. (2004). José Gaos: el transterrado. México: UNAM. 